\title{
Infinite order solutions of complex linear differential equations
}

\author{
Benharrat Belaidi
}




\title{
INFINITE ORDER SOLUTIONS OF COMPLEX LINEAR DIFFERENTIAL EQUATIONS
}

\author{
BENHARRAT BELAIDI
}

Received 16 June, 2004

\begin{abstract}
In this paper we investigate the growth of solutions of the differential equation $f^{(k)}+$ $A_{k-1}(z) f^{(k-1)}+\cdots+A_{1}(z) f^{\prime}+A_{0}(z) f=0$, where $A_{0}(z), \ldots, A_{k-1}(z)$ are entire functions with $0<\sigma\left(A_{0}\right) \leq 1 / 2$. We will show that if there exists a real constant $\beta<\sigma\left(A_{0}\right)$ and a set $E_{\beta} \subset(1,+\infty)$ with $\log \operatorname{dens} E_{\beta}=1$, such that for all $r \in E_{\beta}$, we have $\min _{|z|=r}\left|A_{j}(z)\right| \leq$

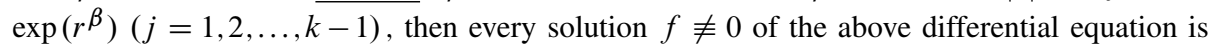
of infinite order with hyper-order $\sigma_{2}(f) \geq \sigma\left(A_{0}\right)$. The paper extends previous results by the author and Hamani.
\end{abstract}

1991 Mathematics Subject Classification: 34M10, 30D35

Keywords: linear differential equations, growth of entire function, hyper-order

\section{INTRODUCTION AND STATEMENT OF MAIN RESULT}

For $k \geq 2$ we consider the linear differential equation

$$
f^{(k)}+A_{k-1}(z) f^{(k-1)}+\cdots+A_{1}(z) f^{\prime}+A_{0}(z) f=0,
$$

where $A_{0}(z), \ldots, A_{k-1}(z)$ are entire functions with $A_{0}(z) \not \equiv 0$. It is well-known that all solutions of equation (1.1) are entire functions and if some of the coefficients of (1.1) are transcendental, then (1.1) has at least one solution with order $\sigma(f)=$ $+\infty$.

Recently, the growth theory of complex differential equations has been an active research area, and the growth problems of linear differential equations have an important aspect in this area. By defining the hyper-order $[9,11]$, the growth of infinite order solutions of differential equations has been more precisely estimated (see $[3,4,9])$. Our starting point for this paper is a result by Kwon in [9]:

Theorem A $([9, \mathrm{p} .489])$. Let $A(z)$ and $B(z)$ be entire functions where $0<$ $\sigma(B)<1 / 2$, and let there exist a real constant $\beta<\sigma(B)$ and a set $E_{\beta} \subset[0,+\infty)$ with $\overline{\operatorname{dens}} E_{\beta}=1$ such that for all $r \in E_{\beta}$, we have

$$
\min _{|z|=r}|A(z)| \leq \exp \left(r^{\beta}\right) .
$$


Then every solution $f \not \equiv 0$ of the linear differential equation

$$
f^{\prime \prime}+A(z) f^{\prime}+B(z) f=0
$$

is of infinite order with hyper-order

$$
\sigma_{2}(f)=\varlimsup_{r \rightarrow+\infty} \frac{\log \log T(r, f)}{\log r} \geq \sigma(B) .
$$

Here the order $\sigma(f)$ of an entire function $f$ is defined by

$$
\sigma(f)=\varlimsup_{r \rightarrow+\infty} \frac{\log T(r, f)}{\log r}=\varlimsup_{r \rightarrow+\infty} \frac{\log \log M(r, f)}{\log r},
$$

where $T(r, f)$ is the Nevanlinna characteristic function of $f$ (see [7]), and $M(r, f)=$ $\max _{|z|=r}|f(z)|$.

This result has been recently generalized to a higher order case (1.1) by the author and Hamani as follows (see [4]):

Theorem B ([4]). Let $A_{0}(z), \ldots, A_{k-1}(z)$ be entire functions with $0<\sigma\left(A_{0}\right)<$ $1 / 2$, and let there exist a real constant $\beta<\sigma\left(A_{0}\right)$ and a set $E_{\beta} \subset[0,+\infty)$ with $\underline{\text { dens }} E_{\beta}=1$ such that for all $r \in E_{\beta}$, we have

$$
\min _{|z|=r}\left|A_{j}(z)\right| \leq \exp \left(r^{\beta}\right) \quad(j=1,2, \ldots, k-1) .
$$

Then every solution $f \not \equiv 0$ of (1.1) is of infinite order with hyper-order

$$
\sigma_{2}(f)=\varlimsup_{r \rightarrow+\infty} \frac{\log \log T(r, f)}{\log r} \geq \sigma\left(A_{0}\right) .
$$

In this paper, we extend Theorem B by proving:

Theorem 1.1. Let $A_{0}(z), \ldots, A_{k-1}(z)$ be entire functions where $0<\sigma\left(A_{0}\right) \leq$ $1 / 2$, and let there exist a real constant $\beta<\sigma\left(A_{0}\right)$ and a set $E_{\beta} \subset(1,+\infty)$ with $\log \operatorname{dens} E_{\beta}=1$ such that for all $r \in E_{\beta}$, we have

$$
\min _{|z|=r}\left|A_{j}(z)\right| \leq \exp \left(r^{\beta}\right) \quad(j=1,2, \ldots, k-1) .
$$

Then every solution $f \not \equiv 0$ of (1.1) is of infinite order with hyper-order

$$
\sigma_{2}(f)=\lim _{r \rightarrow+\infty} \frac{\log \log T(r, f)}{\log r} \geq \sigma\left(A_{0}\right) .
$$

Thus our contribution is to treat the case $\sigma\left(A_{0}\right)=\frac{1}{2}$. The method used in this paper will be quite different from that in the proof of Theorem B (see [4]). The main ingredient in the proof is Lemma 2.2, based on a special case of a theorem of Drasin and Shea [5, Theorem 8.1]. 
Remark 1.1. The conclusions of Theorem 1.1 are also held under the hypothesis $0<\mu\left(A_{0}\right) \leq 1 / 2$, where $\mu(f)$ is the lower order of $f$ defined by

$$
\mu(f)=\varliminf_{r \rightarrow+\infty} \frac{\log T(r, f)}{\log r}=\varliminf_{r \rightarrow+\infty} \frac{\log \log M(r, f)}{\log r} .
$$

To ease the exposition we treat in detail only Theorem 1.1 and in Section 2 we confine ourselves to highlighting the modifications of our proof necessary to obtain the lower order result.

\section{PRELIMINARY LEMMAS}

Our proofs depend mainly upon the following lemmas. Before starting these Lemmas, we recall the concepts of density and logarithmic density of subsets of $(1,+\infty)$. For $E \subset(1,+\infty)$, we define the linear measure of a set $E$ by $\mathrm{m}(E)=$ $\int_{1}^{+\infty} \chi_{E}(t) d t$, where $\chi_{E}$ is the characteristic function of $E$, and define the logarithmic measure of a set $E$ by $\operatorname{lm}(E)=\int_{1}^{+\infty} \frac{\chi_{E}(t)}{t} d t$. The upper density and the upper logarithmic density of $E$ are defined by the formulae

$$
\overline{\operatorname{dens}} E=\varlimsup_{r \rightarrow+\infty} \frac{\mathrm{m}(E \cap[1, r])}{r-1}, \quad \overline{\log \operatorname{dens}} E=\varlimsup_{r \rightarrow+\infty} \frac{\operatorname{lm}(E \cap[1, r])}{\log r} .
$$

The lower density and the lower logarithmic density, dens $E$ and $\log$ dens $E$, are defined similarly with the limit superior replaced by the limit inferior. It is easy to verify $[10$, p. 121] that

$$
0 \leq \underline{\operatorname{dens}} E \leq \underline{\log \operatorname{dens}} E \leq \overline{\log \operatorname{dens}} E \leq \overline{\operatorname{dens}} E \leq 1
$$

for any $E \subset(1,+\infty)$.

For entire $f$, let $L(r, f)=\min _{|z|=r}|f(z)|$. The method of Denjoy-Kjellberg [8, pp. 193-196] shows for entire $f$ with $f(0)=1$ and for $0<\lambda<1$ that there exist positive constants $k_{1}(\lambda)$ and $k_{2}(\lambda)$ such that

$$
\begin{aligned}
\int_{r}^{R}(\log L(t, f)-(\cos \pi \lambda) & \log M(t, f)) \frac{d t}{t^{1+\lambda}} \\
& \geq k_{1}(\lambda) \frac{\log M(r, f)}{r^{\lambda}}-k_{2}(\lambda) \frac{\log M(4 R, f)}{R^{\lambda}}
\end{aligned}
$$

valid for $0<r<R$.

If $\mu(f)<\sigma(f) \leq 1 / 2$, let $\beta$ and $\alpha$ satisfy $\mu(f)<\beta<\alpha<\lambda<\sigma(f) \leq 1 / 2$. Let us choose $r_{m} \rightarrow+\infty$ with $R_{m}>r_{m}$ satisfying $r_{m}^{\lambda}=o\left(\log M\left(r_{m}, f\right)\right)$ and $\log M\left(4 R_{m}, f\right)=o\left(R_{m}^{\lambda}\right)$ to conclude that

$$
\int_{r_{m}}^{R_{m}} \frac{\log L(t, f)}{t^{1+\lambda}} d t \rightarrow \infty
$$


as $m \rightarrow \infty$. Thus there exists $s_{m} \rightarrow \infty$ with

$$
\log L\left(s_{m}, f\right)>s_{m}^{\alpha} .
$$

If $\mu(f)=\sigma(f)<1 / 2$ and $\beta<\sigma(f)$, the classical $\cos \pi \sigma$ theorem (contained in (2.3)) yields $s_{m} \rightarrow \infty$ satisfying (2.4). In both of the above cases results of Barry (see [1, p. 294] and [2, Theorem 4]) also imply the existence of unbounded $s_{m}$ satisfying (2.4).

$$
\text { If } \beta<\mu(f)=\sigma(f)=\frac{1}{2} \text {, then either there exists } s_{m} \rightarrow \infty \text { satisfying }
$$

$$
\log L\left(s_{m}, f\right)>\varepsilon \log M\left(s_{m}, f\right)
$$

for some $\varepsilon>0$ and hence also satisfying (2.4), or

$$
\log L(r, f)=o(\log M(r, f)) .
$$

Lemma 2.1 ([6]). Let $f(z)$ be a nonconstant entire function, and let $\alpha>1$ and $\varepsilon>0$ be given constants. Then the following two statements hold:

(i) There exist a constant $c>0$ and a set $E_{1} \subset[0, \infty)$ having a finite linear measure such that for all $z$ satisfying $|z|=r \notin E_{1}$, we have

$$
\left|\frac{f^{(k)}(z)}{f(z)}\right| \leq c\left[T(\alpha r, f) r^{\varepsilon} \log T(\alpha r, f)\right]^{k} \quad(k \in \mathbb{N}) .
$$

(ii) There exist a constant $c>0$ and a set $E_{2} \subset[0,2 \pi)$ that has linear measure zero, such that if $\psi_{0} \in[0,2 \pi) \backslash E_{2}$, then there is a constant $R_{0}=R_{0}\left(\psi_{0}\right)>1$ such that for all $z$ satisfying $\arg z=\psi_{0}$ and $|z|=r \geq R_{0}$, we have

$$
\left|\frac{f^{(k)}(z)}{f(z)}\right| \leq c[T(\alpha r, f) \log T(\alpha r, f)]^{k} \quad(k \in \mathbb{N}) .
$$

Lemma 2.2 ([5,7]). Suppose that $f(z)$ is entire with $\sigma(f) \leq 1 / 2$ and $\rho<\sigma(f)$. Then either there exists $\left\{r_{m}\right\}$ such that $r_{m} \rightarrow \infty$ and

$$
\min _{|z|=r_{m}} \log |f(z)|>r_{m}^{\rho}
$$

or, if

$$
K_{r}(\rho)=K_{r}=\left\{\theta \in[0,2 \pi]: \log \left|f\left(r e^{i \theta}\right)\right|<r^{\rho}\right\},
$$

there exists a set $E(1) \subset[1,+\infty)$ with $\underline{\log \operatorname{dens}} E(1)=1$ such that for all $r \in E(1)$, $K_{r}$ satisfies

$$
\mathrm{m}\left(K_{r}\right) \rightarrow 0 \quad \text { as } r_{m} \rightarrow \infty
$$




\section{PRoof AND EXAMPLE}

Proof of Theorem 1.1. Let $f \not \equiv 0$ be a solution of (1.1). It follows from (1.1) that

$$
\left|A_{0}(z)\right| \leq\left|\frac{f^{(k)}}{f}\right|+\left|A_{k-1}(z)\right|\left|\frac{f^{(k-1)}}{f}\right|+\cdots+\left|A_{1}(z)\right|\left|\frac{f^{\prime}}{f}\right| .
$$

Let $\beta<\sigma\left(A_{0}\right)$ and suppose that $\beta<\alpha<\sigma\left(A_{0}\right)$ and that there is a set $E_{\beta} \subset(1,+\infty)$ of lower logarithmic density 1 satisfying (1.8). Set

$$
\begin{aligned}
& E=\left\{z:|z|=r \in E_{\beta}\right. \\
& \left.\quad \text { and }\left|A_{j}(z)\right|=\min _{|z|=r}\left|A_{j}(z)\right| \quad(j=1,2, \ldots, k-1)\right\} .
\end{aligned}
$$

Then $\log \operatorname{dens}\{|z|: z \in E\}=1$ and

$$
\left|A_{j}(z)\right| \leq \exp \left(r^{\beta}\right)(j=1,2, \ldots, k-1)
$$

for all $z \in E$.

The proof is divided into two cases depending on the behavior of the minimum modulus of $A_{0}(z)$ on $|z|=r$ by Lemma 2.2. First, we assume that there exists $r_{m}$ such that $r_{m} \rightarrow \infty$ and

$$
\log \left|A_{0}\left(r_{m} e^{i \theta}\right)\right|>r_{m}^{\alpha}
$$

for all $\theta \in[0,2 \pi]$, and for any positive real number $\alpha<\sigma\left(A_{0}\right)$. Furthermore, by Lemma 2.1 (ii), there exists $\theta_{0} \in\left[\beta_{1}, \beta_{2}\right]$ such that if $z=r e^{i \theta_{0}}$,

$$
\left|\frac{f^{(j)}(z)}{f(z)}\right| \leq[T(2 r, f)]^{k+1} \quad(j=1, \ldots, k)
$$

for all sufficiently large $r$. Hence from (3.1), (3.3), (3.4) and (3.5), we have

$$
\exp \left(r_{m}^{\alpha}\right) \leq\left(1+(k-1) \exp \left(r_{m}^{\beta}\right)\right)\left[T\left(2 r_{m}, f\right)\right]^{k+1},
$$

for all sufficiently large $r_{m}$. Therefore $f$ has infinite order and

$$
\sigma_{2}(f)=\varlimsup_{r_{m} \rightarrow+\infty} \frac{\log \log T\left(r_{m}, f\right)}{\log r_{m}} \geq \alpha .
$$

Thus the result of Theorem 1.1 follows since $\alpha$ is arbitrary. Theorem 1.1 is proved in the first case.

Now, we assume that there is a set $E(1)$ with $\underline{\log \operatorname{dens}} E(1)=1$ such that for all $r \in E(1)$, we have

$$
\mathrm{m}\left(K_{r}\right) \rightarrow 0 \text {, as } r \rightarrow \infty,
$$

where

$$
K_{r}=K_{r}(\alpha)=\left\{\theta: \log \left|A_{0}\left(r e^{i \theta}\right)\right|<r^{\alpha}\right\}
$$


By Lemma 2.1 (i) and (3.8), we have a set $E_{1} \subset E$ (1) with $\log$ dens $E_{1}=1$ such that if $z=r e^{i \theta}$

$$
\left|\frac{f^{(j)}(z)}{f(z)}\right| \leq r[T(2 r, f)]^{k+1} \quad(j=1, \ldots, k)
$$

holds for all $r \in E_{1}$, and for all $\theta \in[0,2 \pi]$. Therefore it follows from (3.1), (3.3), (3.9) and (3.10) that

$$
\exp \left(r^{\alpha}\right) \leq\left(1+(k-1) \exp \left(r^{\beta}\right)\right) r[T(2 r, f)]^{k+1},
$$

for all $r \in E_{1} \cap E$, and for all $\theta \in[0,2 \pi] \backslash K_{r}$. Since $[0,2 \pi] \backslash K_{r}$ is nonempty for all sufficiently large $r \in E_{1} \cap E$ by (3.8), we conclude that $f$ has infinite order and

$$
\sigma_{2}(f)=\varlimsup_{r \rightarrow+\infty} \frac{\log \log T(r, f)}{\log r} \geq \alpha .
$$

Thus the result of Theorem 1.1 follows since $\alpha$ is arbitrary. Hence the proof of Theorem 1.1 is now complete.

Next, we give an example which illustrates Theorem 1.1.

Example. Let $P_{1}(z), \ldots, P_{k-1}(z)$ be nonconstant polynomials, and let $h_{1}(z)$, $\ldots, h_{k-1}(z)$ be entire functions satisfying $\sigma\left(h_{j}\right)<\operatorname{deg} P_{j}(j=1, \ldots, k-1)$. Then, by Theorem 1.1 , every solution $f \not \equiv 0$ of the equation

$$
f^{(k)}+h_{k-1}(z) e^{P_{k-1}(z)} f^{(k-1)}+\cdots+h_{1}(z) e^{P_{1}(z)} f^{\prime}+\frac{\sin \sqrt{z}}{\sqrt{z}} f=0
$$

is of infinite order with $\sigma_{2}(f) \geq \sigma\left(\frac{\sin \sqrt{z}}{\sqrt{z}}\right)=\frac{1}{2}$ since

$$
\min _{|z|=r}\left|h_{j}(z) e^{P_{j}(z)}\right| \rightarrow 0 \quad(j=1, \ldots, k-1)
$$

as $r \rightarrow+\infty$.

Remark 3.1. The conclusions of our theorem are also held under the hypothesis $0<\mu\left(A_{0}\right) \leq 1 / 2$. For $\beta<\mu\left(A_{0}\right)$, we first establish as before that either (2.4) holds for some sequence $s_{m} \rightarrow \infty$ or (2.11) holds for a set $E$ (1) of lower logarithmic density 1 . If $\mu\left(A_{0}\right)<1 / 2$, we conclude from Kjellberg's lower order extension [8] of the $\cos \pi \sigma$ theorem (essentially (2.3)) that for each $\beta<\mu\left(A_{0}\right)$ there exists $s_{m} \rightarrow \infty$ satisfying (2.4). Thus we suppose $\mu\left(A_{0}\right)=1 / 2$. Either (2.5) holds for some $s_{m} \rightarrow \infty$ and hence (2.4) also holds, or alternatively (2.6) holds. By the remarks following Theorem 8.1 in [5, p. 283], (2.6) implies the set $K_{r}$ defined in (2.10) satisfies (2.11) for all $r$ in some set $E(1)$ of lower logarithmic density 1. 


\section{REFERENCES}

[1] P. D. Barry, “On a theorem of Besicovitch,” Quart. J. Math. Oxford Ser. (2), vol. 14, pp. 293-302, 1963.

[2] P. D. Barry, "Some theorems related to the $\cos \pi \rho$ theorem," Proc. London Math. Soc. (3), vol. 21, pp. 334-360, 1970 .

[3] B. Belaidi, "Estimation of the hyper-order of entire solutions of complex linear ordinary differential equations whose coefficients are entire functions," Electron. J. Qual. Theory Differ. Equ., no. 5, pp. 1-8, 2002.

[4] B. Belaidi and K. Hamani, "Order and hyper-order of entire solutions of linear differential equations with entire coefficients," Electron. J. Differential Equations, no. 17, pp. 1-12, 2003.

[5] D. Drasin and D. F. Shea, "Convolution inequalities, regular variation and exceptional sets," $J$. Analyse Math., vol. 29, pp. 232-293, 1976.

[6] G. G. Gundersen, "Estimates for the logarithmic derivative of a meromorphic function, plus similar estimates," J. London Math. Soc. (2), vol. 37, no. 1, pp. 88-104, 1988.

[7] S. Hellerstein, J. Miles, and J. Rossi, "On the growth of solutions of certain linear differential equations," Ann. Acad. Sci. Fenn. Ser. A I Math., vol. 17, no. 2, pp. 343-365, 1992.

[8] B. Kjellberg, "On the minimum modulus of entire functions of lower order less than one," Math. Scand., vol. 8, pp. 189-197, 1960.

[9] K.-H. Kwon, "On the growth of entire functions satisfying second order linear differential equations," Bull. Korean Math. Soc., vol. 33, no. 3, pp. 487-496, 1996.

[10] M. Tsuji, Potential theory in modern function theory. Tokyo: Maruzen Co. Ltd., 1959.

[11] C.-C. Yang and H.-X. Yi, Uniqueness theory of meromorphic functions, ser. Mathematics and its Applications. Dordrecht: Kluwer Academic Publishers Group, 2003, vol. 557.

\section{Author's address}

\section{Benharrat Belaidi}

Department of Mathematics, University of Mostaganem, B. P. 227 Mostaganem, Algeria

E-mail address: belaidi@univ-mosta.dz and belaidi.benharratecaramail.com 